\title{
A corporeidade feminina na publicidade: algumas reflexões sobre representações normativas
}

\author{
Cristina Santos \\ Universidade Lusófona de Humanidades e Tecnologias \\ E-mail: cristina.afsantos@gmail.com
}

\begin{abstract}
RESUMO
Com o presente artigo pretende-se reflectir sobre a forma como a corporeidade feminina é representada através da publicidade. Paralelamente, procura-se perceber se o discurso publicitário poderá contribuir para o reforço ou para a ruptura de uma normatividade corporal da mulher, consolidando ou desvanecendo estereóti-

pos femininos. Para o efeito, efectuouse uma revisão da literatura, a qual permitiu detectar a particular preponderância de corpos que correspondem a uma suposta perfeição corporal. Desta forma, o ideal de beleza feminino aparenta assentar numa trilogia que concilia beleza, juventude e magreza.
\end{abstract}

Palavras-chave: corporeidade feminina; publicidade; representações corporais.

\section{Abstract}

With this article we intend to reflect how the female corporeality is represented through advertising. At the same time, we seek to realize if the advertising discourse can strengthen or break a body normativity of women, consolidating or fading stereotypes. For this purpose, it was carried out a literature review, which allowed detecting the particular preponderance of bodies that correspond to an

Data de submissão: 11-05-2016. Data de aprovação: 23-10-2016.

A Revista Estudos em Comunicação é financiada por Fundos FEDER através do Programa Operacional Factores de Competitividade - COMPETE e por Fundos Nacionais através da FCT - Fundação para a Ciência e a Tecnologia no âmbito do projeto Comunicação, Filosofia e Humanidades (LabCom.IFP) UID/CCI/00661/2013. 
alleged bodily perfection. So, the female trilogy that combines beauty, youth and ideal of beauty appears to be based on a thinness.

Keywords: female corporeality; advertising; body representations.

\section{INTRODUÇãO}

$\mathrm{O}$ presente ensaio tem dois objectivos principais: compreender como a corporeidade feminina é representada pela publicidade e perceber até que ponto o discurso publicitário explora uma normatividade corporal genderizada. Sabe-se que o corpo apresenta uma importância crucial para a mulher contemporânea, influenciando as suas vivências, e que a concepção de uma corporeidade feminina perfeita gira em torno de três características: beleza, magreza e juventude. Esta trilogia estética é explorada pela publicidade, cujas personagens personificam, maioritariamente, essa modelação corporal. São apresentados conteúdos padronizados, versões idealizadas da mulher, visualidades hegemónicas, aparências estereotipadas e arquétipos estandardizados. Aparentemente, assiste-se a uma emergente confluência normalizadora, a qual espelha a emergência de uma reflexão e discussão de uma problemática provavelmente enraizada na contemporaneidade.

\section{A CORPOREIDADE NA CONTEMPORANEIDADE}

Se outrora o corpo era usado como um instrumento de trabalho, na presente sociedade este é encarado como um objecto valioso, a ser trabalhado, 'mimado' e exibido, dado o poder que a corporeidade e a visualidade possuem na contemporaneidade. Assiste-se a uma hiperbolização da aparência, centrada na exibição e gestão de impressões, dada a tendência para correlacionar o aspecto exterior com a popularidade dos indivíduos (Maffesoli, 1990; Featherstone, 1991, 1994; Drotner, citada por Boëthius, 1995; Pais, 1995; Campbell, 2004; Alvarado, 2006; Fonseca, 2007; Dittmar, 2008; Veríssimo, 2008; Fehr, 2009; Januário e Cascais, 2012). A imagem dos sujeitos surge, pois, como um importante factor na avaliação que as pessoas efectuam umas das outras, sendo essa apreciação tanto mais positiva quanto maior a aproximação aos ideais de beleza vigentes (Pereira et al., 2011). 
Como consequência, existe uma propensão para o culto do corpo, que tende a ir ao encontro dos cânones estéticos prevalecentes (Henderson e DeLong, 2000; Pais, 2003, 2005; Veríssimo, 2008). Para o efeito, várias práticas corporais são postas em prática, como por exemplo, o consumo de produtos de beleza, a implementação de dietas; a ida a ginásios e a consultas de nutrição, a ingestão de esteróides ou a aposta na cirurgia estética. Como consequência, o corpo é palco de crescentes investimentos (Featherstone, 1991; Henderson e DeLong, 2000; Ferreira, 2004; Pais, 2003, 2005; Dens et al., 2008; Veríssimo, 2008; Dittmar, 2009; Mota-Ribeiro, 2010), dado que “(...) no imaginário corporal contemporâneo, o corpo deixa de ser tomado como um destino herdado ao qual cada um é abandonado (...)" (Ferreira, 2004: 58). Desta forma, a corporeidade torna-se numa realidade passível de opções e de escolhas, assistindo-se à respectiva emancipação “(...) relativamente à postura naturalista que o toma como mero adquirido, realidade geneticamente herdada, governada por processos biológicos incontornáveis" (Ferreira, 2004: 58).

\section{A IMPORTÂNCIA DA CORPOREIDADE EM CONTEXTO FEMININO}

Se a importância da corporeidade é transversal à sociedade, a problemática ganha um particular destaque em contexto feminino. A aparência é mais valorizada e associada à mulher, a qual aparenta concentrar mais as suas vivências em torno da própria fisionomia, que exercerá uma maior influência na forma como se auto-percepciona, mas também no modo como os outros a encaram. É ao sexo feminino que é exigido um maior cuidado e aprumo com o seu aspecto físico, sendo este, inclusive, a principal propriedade que define uma 'mulher interessante' (Pais, 1995). Mota-Ribeiro (2010) considera que a mulher que não cuidar do seu visual poderá não corresponder ao papel que supostamente deveria desempenhar, pelo que deve evitar "(...) ser vista como não atraente" (2010: 140), facto que ganha um maior protagonismo dada a visibilidade feminina: “(...) as mulheres têm a consciência de estar sempre em palco (...). Por isso, é necessário vigiar constantemente a apresentação da sua personagem em cena" (2010: 141). Neste enquadramento, o espelho desempenha um papel relevante nessa monitorização, dada a sua utilidade, pois o facto de ser encarado como um símbolo de feminilidade não se deve a uma 
pressuposta vaidade, mas pela auto-vigilância que a mulher executa (Berger, 1972, citado por Mota-Ribeiro, 2010).

Vários outros indicadores ilustram essa aparente prevalência feminina. Ollila (2008) (citada por Autio et al., 2013) constatou que para as raparigas o aspecto visual contribui para o sucesso juvenil. Na opinião destas jovens, é importante, para o sexo feminino, ter uma imagem cuidada. Rodrigues (2013) assinala que os seus entrevistados mencionam que as mulheres são mais centradas na fisionomia do que os homens. É mencionada a paciência feminina, percepcionada como sendo ilimitada, para com as rotinas de cuidados corporais mais exigentes em termos de tempo. Também na compra de vestuário, os participantes na pesquisa da investigadora consideraram as mulheres mais indecisas e demoradas. Aliás, a roupa aparenta ser um bem particularmente associado ao universo feminino, já que as mulheres valorizam e interessam-se mais pela indumentária, para além de apresentarem um maior envolvimento na respectiva aquisição (Corrigan, 1997; Veríssimo, 2008). A este propósito, assinalemos a maior apetência aquisitiva feminina, dada a sua privilegiada ligação ao consumo (Ganetz, 1995; Cardoso e Pinto, 2009; Deutsch e Theodorou, 2010; Ribeiro, 2010). Num inquérito realizado à população lisboeta, Pais (1995) detectou que um dos temas mediáticos com maior interesse para os jovens é a imagem e cuidados com o corpo, sendo mais significativo junto do público feminino. Desta forma, culturalmente a mulher está mais predisposta para a valorização da aparência, interiorizando essa ligação como algo 'natural' e que faz, inclusive, parte da sua identidade: “(...) women are (...)encouraged to believe it is important (...)to look good in order to be valued" (Dens et al., 2008: 417). Este panorama poderá contextualizar o facto de Brinkman et al. (2012) terem detectado que algumas das crianças participantes na sua investigação afirmarem ser conhecidas por desafiarem normatividades femininas associadas a uma expectável visualidade: “(...) one girl described (...) she was seen as being not very feminine and that people expected her to always conform to that identity" (Brinkman et al., 2012: 8).

A proeminente relevância corporal feminina poderá justificar o facto de o corpo ser um assunto mais sensível e problemático para o sexo feminino (Strandbu e Kvalem, 2014). Diversas pesquisas indicam que a mulher, comparativamente com o homem, apresenta maiores preocupações e é mais exigente e ansiosa com o seu aspecto físico, monitorizando, de forma mais vincada, o seu visual; que tende a ser mais insatisfeita com a sua imagem corporal e 
revela uma maior propensão para práticas que lhe permite melhorar a sua aparência (Ferreira, 2003; Holdorf, 2005; Veríssimo, 2008; Ashikali e Dittmar, 2012; Vilhjalmsson et al., 2012; Autio et al., 2013), como praticar exercício físico, iniciar uma dieta restritiva, usar cosméticos, depilar-se, usar roupas justas, esticar ou encaracolar o cabelo, fazer cirurgia estética ou lipo-aspiração. Este conjunto de práticas corresponde a uma 'disciplina feminina do corpo', que têm como principal objectivo a modelação ou transformação do espaço corporal feminino no sentido de se aproximarem de um modelo estandardizado de feminilidade (Magalhães, 2011).

Ciente da preponderância das práticas corporais para o sexo feminino, Pais (1996) recorre a uma analogia, numa equiparação de rituais: “(...) os ritos de beleza, para algumas mulheres, têm substituído os ritos religiosos ou adquirido um configuração de natureza parareligiosas: há verdadeiras cruzadas pelo controlo de peso; a comida e os doces são uma tentação a que se procura fugir; os jejuns religiosos dão lugar aos jejuns dietéticos; os regimes alimentares são objecto de crenças, de mistificações, de fé; aos esteticistas confessam-se as angústias das rugas, borbulhas e flacidez da pele; os Evangelhos são substituídos pelas revistas de beleza ("siga religiosamente as instruções"...); as balanças são os actuais confessionários de pecados alimentares; os ginásios são os locais de culto desse "deus" que nos reveste e é o nosso corpo e através do qual se protagoniza a sexualidade" (Pais, 2006: 4). Através desta "caricatura', o investigador mostra a relevância que a aparência tem para a mulher e o impacto que essa preponderância apresenta na sua vivência quotidiana.

A ligação existente entre o sentimento de satisfação com o corpo (autoestima corporal) e o de satisfação enquanto pessoa (auto-estima pessoal) indicia que as raparigas têm maiores probabilidades de possuírem baixos níveis de auto-estima, tal como dá conta a literatura (Ferreira, 2003; Dittmar, 2009; Ashikali e Dittmar, 2012; Dens et al., 2008; Vilhjalmsson et al., 2012; Chang et al., 2014). Ferreira (2003) frisa que a auto-estima identitária negativa é mais relevante nas mulheres; Dens et al. (2008) assinalam que é o sexo masculino que tem uma auto-estima mais alta; Pereira e Antunes (2008) realçam que a mulher possui um auto-conceito menos positivo e Silva (2011) detectou que, na Europa, as pessoas que vivem com um maior nível de bem-estar subjectivo 
(BES) ${ }^{1}$ são os indivíduos masculinos. Porém, a idade da mulher aparenta ser determinante. Twigg afirma que pesquisas recentes sugerem que "(...)older women are in fact happier with their bodies and appearance than younger ones (...)" (2009: 11). A autora avança com uma possível razão para este aparente paradoxo: "(...)the increasingly heavy pressure placed on younger women (...).Older women may be more satisfied because they are no longer subject to these stringent norms of femininity" (2009: 11-12).

Fannon (2016) quis conhecer as experiências de mulheres com deficiência visual relativamente aos ideais de beleza e verificou que não só tinham uma noção clara de uma suposta corporeidade ideal (corpo magro, bonito e jovem), como demonstraram preocupar-se com a questão estética: "Not unlike sighted women they too are acutely aware of the significance placed on female appearance; they feel pressure to meet feminine beauty norms; they work hard on their own appearance, using diet, fitness, fashion and grooming, to look and feel attractive; and they too wish to change their appearance in response to personal dissatisfaction about the shape and look of their bodies" (Fannon: 2016: $\mathrm{s} / \mathrm{p}^{2}$ ). A investigadora constatou, igualmente, que estas mulheres acreditam que o 'desvio' das normas visuais tem impactos pessoais e sociais, podendo fazer a diferença na obtenção de emprego; de ser promovida; de arranjar amigas ou um parceiro. Acrescente-se que os rituais de beleza e as práticas de gestão da aparência que desenvolvem poderão servir como um suporte importante para a inclusão, fazendo com que as mulheres portadoras de deficiência se sintam femininas e em igualdade entre os seus pares. No entanto, consideram que têm que dedicar uma maior atenção à sua aparência, comparativamente com as mulheres sem qualquer deficiência, pois consideram que só assim conseguirão transmitir a imagem de pessoas capazes e independentes. Por outro lado, a incapacidade para retribuir visualmente os olhares dos outros, para avaliar a sua aparência com facilidade e rapidez ou medir as reacções de outras pessoas quanto à imagem que transmitem são questões que poderão desencadear sentimentos de vulnerabilidade e de insegurança.

1. O BES traduz as respostas emocionais dos indivíduos nos seguintes domínios: satisfação com a vida, felicidade, saúde e relações interpessoais, para além das avaliações subjectivas relativamente à governação e à sociedade (Silva, 2011).

2. O artigo de onde foi retirada a citação encontra-se apenas disponível em formato HTLM, em 'texto corrido', sem qualquer paginação: http://dsq-sds.org/article/view/ $1366 / 1538$. 
Se a mulher é mais focada na sua aparência, esta particular incidência é justificada com base nos mais diversos argumentos. Segundo o testemunho de diversos autores, como Corrigan (1997) ou Pais (2003), os diferentes momentos históricos afectos ao género feminino acabam por determinar essa maior predisposição. Devido ao facto de as mulheres terem ocupado, historicamente, posições sociais menos reconhecidas e sedimentadas no costume, a moda permitia a conciliação entre o desejo de imitação geral com o de distinção, refere Simmel (1969, 1991, 2004). Por sua vez, Ganetz (1995) realça que, como a mulher foi sempre menos socialmente visível do que o homem, a moda ajudou a ultrapassar essa barreira sexista. Já Corrigan (1997) menciona o facto de o surgimento das lojas ter proporcionado uma escapatória à esfera doméstica feminina, concedendo à mulher um novo espaço no domínio público. Se antes da Revolução Industrial eram os homens quem mais investiam na sua imagem, por terem que rivalizar entre si para conquistarem as mulheres, é na alvorada da Revolução Industrial que o universo feminino adopta uma cultura galante, investindo na imagem e na moda ${ }^{3}$, menciona Pais (2003). Tal como no caso feminino, também o poder que a estetização e a visualidade apresentam no contexto juvenil é inequívoco (Gonçalves, 1995; Lehmann et al., 1998; Silva, 2001; Pais, 2003, 2005; Brull e Abeja, 2006; Nunes, 2007; Campos, 2010), inclusive para o desenvolvimento da respectiva auto-estima (Daters, 1996).

\section{OS DENOMINADORES DE UMA PERFEIÇÃO CORPORAL FEMININA}

A concepção de corpo ideal feminino está alocado a três eixos principais: beleza, magreza e juventude. A corporeidade perfeita assenta, desta forma, numa trilogia, que reflecte os ideais de beleza ocidentais e contemporâneos,

3. Esse investimento devia-se ao modelo de matrimónio adoptado na Idade Média, na Europa Ocidental, o qual assentava numa forte redução da nupcialidade e num adiamento da idade da contracção do casamento, para além de sacralizar o celibato e a virgindade feminina. Como consequência, as noivas escasseavam. Factor agravado por fenómenos à época recorrentes como o infanticídio e a poligamia. Um 'bom casamento' era aquele que dava direito a aceder à possessão de terras. Este modelo de matrimónio entra em declínio na alvorada da Revolução Industrial. Nesta fase, o emprego ganha um valor superior à terra. A competição masculina deixa de se centrar na união, mas na posse de um trabalho estável, pelo que os investimentos na indumentária são substituídos pelas qualificações educativas ou profissionais. Assim, as mulheres aspiram a relacionar-se com um jovem detentor de um 'bom emprego', passando a serem as próprias a preocuparem-se mais com a imagem (Pais, 2003). 
os quais são encarados como 'um capital' pessoal a conquistar e a manter. Debrucemo-nos, individualmente, sobre cada um dos três conceitos. Comecemos pelo enunciado primeiramente. A beleza corporal tem vindo a adquirir um crescente valor simbólico (Maffesoli, 1990; Featherstone, 1991, 1994; Drotner, citada por Boëthius, 1995; Campbell, 2004; Fonseca, 2007; Veríssimo, 2008), particularmente para a mulher, cuja conceptualização passa pela posse de um corpo bonito (Mota-Ribeiro, 2010). Pereira e Antunes (2008); Deutsch e Theodorou (2010) e Marion e Nairn (2011) detectaram que à feminilidade se associa a beleza.

Chan (2008), por sua vez, apurou que a ambição de ser bonita foi somente realçada pelas raparigas participantes na sua pesquisa, enquanto Ollila (2008) (citada por Autio et al., 2013) constatou que é relevante, para o sexo masculino, ter uma companheira bonita. Mota-Ribeiro reforça estes resultados, ao argumentar que a "(...) aparência física continua a ser mais determinante na escolha do parceiro para os homens do que para as mulheres" (2010: 152). Aliás esta centralidade ganha contornos deterministas, ao ponto de a própria feminilidade basear-se no atributo beleza, já que, quanto mais bonita for a mulher, mais percepcionada ela é como sendo feminina, processo que não ocorre na esfera masculina. Como perspectiva Mota-Ribeiro (2010), a beleza é uma característica contruída e atribuída socialmente à mulher, já que nada há de fisicamente inerente, de biológico, que torne a aparência feminina mais relevante do que a masculina.

No que concerne à magreza, verifica-se uma “(...) maior severidade para com a obesidade feminina" (Mota-Ribeiro, 2010: 152). O corpo magro é valorizado, ao contrário da gordura, a qual é associada à doença e à falta de controle, de disciplina e de vontade (Andrade, 2002, citada por Palacios, 2004; Mota-Ribeiro, 2010; Pereira et al., 2011; Januário e Cascais, 2012): "Tradicionalmente, o excesso de carne, os corpos volumosos ou fortes são conotados com masculinidade" (Mota-Ribeiro, 2010: 328). Actualmente, por exemplo, a uma silhueta esguia é associado reconhecimento, estatuto, sucesso pessoal, profissional e social (Agnew, 1984). Há várias implicações quando está em causa a obesidade na mulher, nomeadamente uma associação entre a perda de peso e a felicidade e uma relação entre o excesso de peso e a impossibilidade de ser encarada como atraente e de ter uma vida sexualmente activa (Mota-Ribeiro, 2010). Disso dá conta a investigação de Nielsen e Rudberg, a propósito de uma das jovens participantes na sua investigação: “(...) the sur- 
plus fat not only turns Eva into a failure as a sex object, but also indicates that she is a weak and worthless person" (1993: 46). São comuns as mensagens publicitárias em que se demonstra uma efectiva necessidade de as raparigas se tornarem apelativas fisicamente, se apresentarem disponíveis para serem olhadas e, supostamente, escolhidas por um parceiro (Magalhães, 2011).

Quanto à juventude, é almejado "(...) um corpo perfeito que não se degrada com a idade (...)" (Pereira et al., 2011: 162) e ambicionado "(..) manter a imagem corporal de um adolescente (...)" (Pereira et al., 2011: 162). O corpo jovem possui um valor e legitimidade social superior, surgindo, por isso, enquanto corporeidade modal ${ }^{4}$ (Ferreira, 2004, 2011). Para o efeito, concebem-se bens que materializam essa pretensão, implantando-se “(...)la tiranía indiscutible de milagrosos productos y de formulas mágicas capaces de prevenir el envejecimiento y de recuperar la belleza perdida a través del paso irreductible de los años" (Alvarado, 2006: 299). Alvarado (2006) afirma que, mais do que um mito, a busca da eterna juventude constitui, actualmente, uma realidade que mantém as sociedades apegadas à falácia da involução. Instituiu-se que os indivíduos podem enfrentar, com êxito, o irreversível efeito da evolução biológica.

De acordo com Veríssimo (2008), é o corpo feminino que repousa, particularmente, numa tríade do corpo perfeito: a juventude, a beleza e a saúde. Estas características representam as três condições culturais para a fecundidade e a perpetuação da espécie. Curiosamente, e a este propósito, Lipovetsky (2000) (citado por Mota-Ribeiro, 2010) refere que a actual apreciação da magreza da mulher faz eco de determinadas transformações sociais, na medida em que se assiste ao enfraquecimento da associação do corpo feminino à maternidade, consequência do decréscimo do valor social de ser mãe e à valorização da mulher activa e independente. É que o corpo feminino robusto era associado à reprodução (Magalhães, 2011). Já em 1995, Pais detectara essa tendência na população nacional: (...) entre as mulheres, o modelo da maternidade parece já estar cedendo lugar ao modelo da beldade. O ideal da mulher, provavelmente, já não é ter filhos e cuidar deles, numa lógica subordinada à domesticidade, mas fazer tudo para se manter bela" (Pais, 1996: 4).

4. A corporeidade modal é composta por um conjunto de traços e usos corporais socialmente mais valorizados e legitimados que outros (Ferreira, 2004, 2011). 
Acrescente-se que a publicidade contribui para a particular relevância detida pela corporeidade em contexto feminino, por explorar a importância que a aparência pode assumir nas suas vivências (Veríssimo, 2008; Mota-Ribeiro, 2010; Magalhães, 2011), facto que ganha uma maior dimensão quando existe uma relação privilegiada estabelecida pela mulher com a publicidade (Cardoso e Pinto, 2009; Crymble, 2012). Talvez por esse motivo, Magalhães (2014), na análise efectuada aos anúncios publicitários de uma revista feminina e outra masculina, verificou que a publicidade se encontrava em maior número na publicação destinada às mulheres. Aliás, uma das acusações apontadas às marcas e à publicidade decorre do facto de ambas reduzirem as mulheres à aparência. É que a noção ocidentalmente moderna de feminilidade ideal poderá ser entendida como um mecanismo normalizador que oprime o poder feminino, ao circunscrever as mulheres ao seu aspecto exterior (Fannon, 2016).

\section{A REPRESENTAÇÃo CORPORAL FEMININA NO DISCURSO PUbLICITÁ- RIO}

A concepção de uma corporeidade feminina ideal encontra-se presente na publicidade, ao ser dado um maior protagonismo a corpos belos, magros e jovens, já que as personagens que surgem nos anúncios publicitários aparentam materializar essa trilogia corporal. Vejamos de que forma. A beleza constitui um dos traços mais marcantes e transversais das imagens publicitárias (MotaRibeiro, 2003; Araújo, 2013). Veríssimo (2008) assinala que nos anúncios publicitários que analisou evidenciou-se a beleza e a sensualidade das personagens femininas, características que coabitam recorrentemente. Por sua vez, Mota-Ribeiro (2010) identificou expressões e poses femininas com um carácter erótico em que, por vezes, as roupas usadas pelas personagens davam destaque a partes do corpo consideradas essencialmente femininas, como os seios e as pernas. A pesquisa de Magalhães (2011) permitiu verificar que a construção discursiva 'Tornar-se sexy!' emerge sobretudo na publicidade direccionada às raparigas e traduz uma tendência actual de sexualização dos conteúdos e discursos mediáticos. Pons (2006) enumera diversas tipologias femininas presentes no discurso publicitário e que exemplificam, de alguma forma, essa ligação feminina à volúpia: a vampe, a femme enfant ou a pin up. 
A idade, por sua vez, é uma das variáveis mais homogéneas no discurso publicitário, já que a juventude é predominante, enquanto, contrariamente, se verifica a fraca expressividade da velhice (Rosales, 2002; Veríssimo, 2008; Mota-Ribeiro, 2010). A juventude talvez seja uma das maiores promessas que a publicidade faz, surgindo como um signo de narcisismo, aventura, diversão e sedução (Medeiros, 2004). Segundo Ferreira (2011), a publicidade capitaliza o corpo jovem, associando-o ao perfil de corporeidade perfeita. Esse corpo é, pois, fetichizado, cobiçado, obstinadamente desejado e mercantilizado, servindo de suporte figurativo a marcas e produtos. É estabelecida a ideia da perduração da juventude, crença alimentada pelas promessas mercantis da juvenilização dos corpos. De acordo com o investigador, a sociedade encontra-se contaminada “(...) por um complexo de Peter Pan profundamente enraizado" (2011: 260).

Também o peso da mulher parece ser um critério determinante no discurso publicitário (Mota-Ribeiro, 2003): “(...)the "body perfect"ideal is ultra-thin, and whose media models are typically underweight (...) (Dittmar, 2009: 2). Trata-se de uma visualidade que tem várias consequências. Queiroz e Otta (2000) (citados por Palacios, 2004) alegam ser comum que as mulheres se vejam acima do peso, mesmo quando efetivamente tal percepção não corresponde à realidade. De igual forma, a pesquisa de Cunha (2004) (citada por Ferreira, 2011) identificou a existência de uma sobrevalorização do peso real por parte das raparigas inquiridas. Poderá estar em causa uma distorcida concepção feminina de beleza (Ribeiro, 2011), situação para a qual a publicidade poderá contribuir: “(...)images of thin women (...)negatively impated mood and bodily perceptions (...)" (Dens et al., 2008: 415); “(...)exposure to thinideal images is an immediate cause of women's body dissatisfaction (...)" (Ashikali e Dittmar, 2012: 514). De acordo com Lysardo Dias (citada por Ribeiro, 2011), os anúncios publicitários têm vindo a explorar, fundamentalmente, dois tipos de estereótipos femininos, os quais se inter-relacionam: a beleza associada à magreza e a juventude à felicidade. Lipovetsky (2000) (citado por Mota-Ribeiro, 2010) afirma que existem duas normas que dominam a esfera feminina: o anti-peso e o anti-envelhecimento.

As personagens dos anúncios publicitários não são, por norma, uma representação realista, mas uma referência imaginária e idealizada, fazendo eco de padrões estéticos estereotipadamente ideais: "A pessoa ideal surge assim como uma metáfora, uma imagem mítica e mistificadora, representando os 
ícones contemporâneos aceites socialmente (...)" (Veríssimo, 2008: 83). As personagens são colocadas nos anúncios publicitários para terem um efeito de modelagem, ao suportarem a transformação do produto em símbolo. A aparência desses intervenientes transporta valores e atitudes com os quais o consumidor se identifica ou aspira atingir. Os corpos representados na imagem publicitária são, maioritariamente, signos de desejo, principalmente por uma motivação de natureza aspiracional (Medeiros, 2004; Pereira e Veríssimo, 2004; Veríssimo, 2008). Verifica-se a proliferação de "Personajes estereotipados que encarnan un prototipo (...)de feminidad de acuerdo con los ideales de belleza (...)" (Pons, 2006: 309-310), apostando-se, para o efeito, em modelos criados pela indústria de Hollywood. Prevalece a “(...) incorporação (...) de modelos de corporeidade ideal veiculada mediaticamente (...)" (Ferreira, 2003: 276). Diversas pesquisas comprovam essa tendência, dado que as celebridades, os manequins e os ídolos que surgem nos anúncios publicitários, muitos dos quais são ícones de beleza, são frequentemente encarados como uma referência (Ferreira, 2003; Dens et al., 2008; Pereira e Antunes, 2008).

Veríssimo constatou, na publicidade analisada, que esta ferramenta de comunicação “(...) não exterioriza um corpo qualquer, mas um cânone que "apela" a que cada uma das pessoas obtenha e assuma o corpo, não o seu, mas aquele que se vê nas imagens" (2008: 188). Como perspectiva o investigador, "A aceitação desta premissa acaba por "implicar" a negação do próprio corpo" (Veríssimo, 2008: 188). Saliente-se que uma eventual discrepância entre as duas imagens (a detida pelo consumidor e a projectada pela personagem) poderá originar problemas das mais diversas índoles, como a obesidade; a anorexia nervosa ou a bulimia; as frustrações; as depressões; a desilusão; a vergonha; uma baixa auto-estima; os conflitos familiares; a dependência de estupefacientes ou as fobias sociais (Bessenoff, 2006; Dens et al., 2008; Veríssimo, 2008; Dittmar, 2009). Ashikali e Dittmar (2012) fazem referência a essa problemática: "Body dissatisfaction (...)has been identified as a potent and consistent precursor of a range of mental and physical health problems (Ashikali e Dittmar, 2012: 514). Até porque, "Body image has emerged as a core aspect of mental and physical well-being (Dittmar, 2009: 1).

A literatura sublinha que as imagens idealizadas que surgem nos anúncios publicitários afectam a forma como os consumidores se auto-percepcionam (Dens et al., 2008). Ashikali e Dittmar mencionam que inúmeros estudos chegaram à mesma conclusão: “(...)the more time spent watching television 
and reading magazines, the higher the experience of body dissatisfaction" (2012: 515). A publicidade poderá contribuir, de alguma forma, para a já mencionada insatisfação física feminina, dadas as representações da corporeidade que veicula, assentes, maioritariamente, numa mesma referência estética. No entanto, numa pesquisa nacional, a maioria das entrevistadas afirmou que as imagens de mulheres 'ideais' presentes nos anúncios publicitários não tinham quaisquer efeitos ou mesmo que eram benéficas para as mulheres 'reais', já que a beleza pode provocar sensações positivas (Pinto-Coelho e Mota-Ribeiro, 2007). Não obstante a cultura consumista ter como um dos seus ideais o corpo perfeito, o qual é projectado e promovido pela publicidade, poderá haver, igualmente, uma pressão da família e dos pares para que o indivíduo tenha uma silhueta estandardizada, o que potenciará, eventualmente, sentimentos de insatisfação física (Ferreira, 2003; Dens et al., 2008; Ashikali e Dittmar, 2012). A exposição a este tipo de modelos corporais começa numa tenra idade e através dos brinquedos: "Body perfect"ideals are communicated (...)through dolls such as Barbie, and girls as young as 5 to 7 years reported lower body esteem and a greater desire for a thinner body directly after exposure to such doll images dissatisfaction" (Dittmar, 2009: 2). De facto, e ao longo da sua existência, a Barbie, que tem sido um símbolo e um ícone da beleza feminina, foi alvo de descontentamento, acusações e críticas, devido ao irrealismo do seu corpo. Assim, a Mattel alterou a sua estratégia e, no início do ano, procurou "reflectir melhor o mundo que as meninas vêem à sua volta" 5 , tendo sentido necessidade de reinventar os seus produtos, por forma a adaptarem-se à actualidade e a dar resposta às reivindicações da sociedade. No total, lançou trinta e três novas bonecas, na linha Fashionistas, com quatro tipos de corpo ${ }^{6}$.

É devido a esse maior protagonismo da corporeidade feminina que a publicidade a cosméticos é, maioritariamente, direccionada às mulheres, escrevem Rosales (2002); Alvarado (2006); Pereira e Antunes (2008) e Magalhães (2014). Veríssimo (2008) concluiu que, em Portugal, e de acordo com os resultados da sua pesquisa, o mercado de produtos relacionados com o corpo é maioritariamente dirigido à mulher. Nessa argumentação publicitária, dominam as mensagens assentes na beleza, invocando a perfeição física, a qual

5. Sara Marçal, responsável de marketing da Mattel Portugal.

6. Estes modelos poderão ser consultados em: www.barbie.com/en-us. 
poderá ser obtida através dos bens anunciados, particularmente através dos produtos para o tratamento do rosto e de fragrâncias. Lazar (2012) explora a forma como a beleza é metaforicamente conceptualizada na publicidade como uma luta. Através da metáfora conceptual 'embelezamento é guerra' são identificados, nos anúncios publicitários analisados, três dimensões: os problemas (os fenómenos que afectam, negativamente, o alcance de um corpo belo são considerados inimigos. Nesse sentido, o tempo, as agressões ambientais, como a poluição, ou o envelhecimento são considerados adversários femininos); as consumidoras (os corpos das mulheres são campos de batalha e as mulheres são combatentes) e as soluções (em que as marcas surgem como poderosas aliadas nessa luta, ao desenharem estratégias, com o intuito de apoiarem e ajudarem as mulheres nos seus esforços para alcançar a beleza corporal. Determinados produtos são apresentados como armamento 'topo de gama' na 'frente de combate'). Na associação entre guerra e beleza, poderá estar em causa uma abordagem menos positiva, uma vez que o campo de batalha é o corpo da mulher, encarado como um potencial adversário, o que poderá conduzir a uma identidade feminina eventualmente fracturada e à alienação da relação das mulheres com a sua corporeidade.

A publicidade é percepcionada como sendo perpassada por representações estereotipadas relativamente ao género (Jorge, 2014). Constata-se a permanência de diversos estereótipos no discurso publicitário, tal como ilustrado por Medeiros: “(...) são normalmente mãos femininas que nos anúncios lavam, cozinham e arrumam a casa (...)" (2004: 82). Constatação corroborada pela pesquisa de Rosales (2002). Medeiros justifica esta prevalência: “(...) também porque são ainda as mulheres as principais responsáveis pelas tarefas domésticas e pelas compras do lar" (2004: 82). Para Mota-Ribeiro (2010), esta variável de comunicação deveria afastar-se de protótipos femininos idealizados pela sociedade ocidental, de modelos de feminilidade centrados em representações irreais e idealizadas do corpo feminino e promover a divulgação de anúncios publicitários que mostrem “(...) as mulheres como realmente são, não as essencializando, e não as cristalizando em papéis estereotipados" (2010: 63). A investigação da autora, tal como a de Pinto-Coelho (2007) e a de Magalhães (2014), demonstram que a mulher que surge na publicidade é, maioritariamente, bela, jovem e magra, correspondendo ou construindo o ideal de beleza corporal feminino dominante na nossa sociedade. Evidenciase, por isso, a homogeneização da aparência das personagens femininas. São 
várias as marcas presentes em anúncios publicitários que pretendem passar a imagem do que é adequado e normal numa rapariga (Magalhães, 2011).

Esta supremacia visual, que encerra uma suposta perfeição corporal, é ainda ilustrada através do resultado da pesquisa de Mota-Ribeiro (2003). De um total de cento e nove anúncios publicitários de imprensa recolhidos das mais diversas marcas, a investigadora sublinha que apenas em três dessas imagens figuram mulheres teoricamente 'comuns', isto é, não representativas do tipo de fisionomia idealizada. Magalhães (2014) apurou que a maioria das personagens que surgiam nos anúncios publicitários analisados era magra, caucasiana e com cabelo liso, propriedades correspondentes aos estereótipos de ideais de beleza ocidentais ${ }^{7}$. A investigadora ironiza a valorização desse imagem normativa: "Estão assim vedadas possibilidades de sucesso a adolescentes e mulheres que se distanciem do ideal de mulher jovem, branca, magra, ocidental, heterossexual, classe média-alta, não portadora de deficiência" (Magalhães, 2011: 153). Existem, nesse sentido, pressões e sanções para quem não se adeque à norma estética em vigor (Mota-Ribeiro, 2010).

Como consequência, as mulheres que não se enquadram num padrão alocado a um pressuposto corpo belo, magro e jovem tornam-se 'invisíveis'. As 'imperfeições' corporais, dissidentes dos ideais de beleza, encontram-se praticamente ausentes do discurso publicitário. Constata-se a “(...) omissão social do corpo do deficiente, do idoso e do doente (...). Devido às suas enfermidades, que podem causar nos outros sensações desagradáveis, os seus corpos tendem a ser colocados à distância dos demais e até ignorados" (Veríssimo, 2008: 66). A publicidade apresenta “(...) o lado «cor-de-rosa» da vida (...)" (Pereira e Veríssimo, 2004: 183), explorando, precisamente, a positividade, dissipando as fragilidades do consumidor e exacerbando o seu lado narcísico. As dificuldades quotidianas e as restantes “(...) facetas negativas são atreladas ao não-ter o produto" (Camargo, 2013: 22), já que o discurso publicitário é maioritariamente simbólico, ao apresentar os bens como ícones, carregados de significados intangíveis (Santos, 2013). Na transmissão das suas mensagens, a publicidade constrói 'não-realidades', ignorando os aspectos negativos da sociedade, ao criar uma encenação bela e feliz (Veríssimo, 2008). Daí a linguagem publicitária operar enquanto uma vendedora de sonhos, como uma

7. Januário e Cascais (2012) colocam em evidência alguns padrões estéticos ocidentalmente apreciados, como a magreza; as formas rectilíneas; os tons de pele clara e os cabelos lisos. 
fábula e fantasia (Gay et al., 1997; Silva et al., 2011). Tal como poderá ilustrar a investigação desenvolvida por Yönet, Engin e Öztürk (2006) (citada por Pereira et al., 2011), os quais encontraram evidências empíricas de que, embora o sexo feminino afirme que quer ver mulheres comuns na publicidade, quando lhes é pedido para assinalarem as suas preferências de beleza, são escolhidas modelos ideais, em detrimento das 'reais'.

Mas não são somente as pessoas que não sejam bonitas, magras e jovens que se encontram, de alguma forma, menos presentes no discurso publicitário. De facto, a publicidade não explora, por norma, sectores 'minoritários' da sociedade (Medeiros, 2004), denotando-se a maior visibilidade de personagens caucasianas e de casais heterossexuais (Rosales, 2002; Mota-Ribeiro, 2010; Pinto-Coelho e Mota-Ribeiro, 2012). Hahn (1987) (citado por Haller e Ralph, 2001) argumenta que, ao se colocar ênfase na beleza e perfeição corporais, a publicidade exclui as pessoas com deficiência. Como são indivíduos que não se enquadram num padrão alocado a um pressuposto corpo belo, são ignorados pelo discurso publicitário. Porém, pontualmente, a publicidade utiliza corpos alvo de estigmatização para passar, igualmente, a mensagem, desafiando, dessa forma, as visibilidades dominantes, resistindo a uma estética normativa, como aconteceu, por exemplo, com a Desigual e a Dove.

A marca espanhola foi a primeira a escolher uma manequim com vitiligo ${ }^{8}$ para comunicar os seus produtos. Winnie Harlow foi seleccionada para ser a cara da colecção de Outono/Inverno transacta da Desigual. Em comunicado, a marca explicou a motivação da sua escolha, que recaíu sobre uma mulher com uma imagem incomum: "Desafiar os convencionalismos e questionar a normalidade" ${ }^{9}$, objectivo que espelha a própria estratégia e posicionamento da marca. Já a Dove, ao invés de recorrer a personagens com corpos que correspondem aos arquétipos corporais vigentes, que, no entender da marca, reflectem uma definição de beleza restrita e limitada, apostou em testemunhos de mulheres 'comuns', de diversas etnias, variadas faixas etárias e com corpos distintos entre si, numa clara alusão à diversidade corporal. Poderá estar em causa uma tipologia pouco convencional, uma vez que as intervenientes nos anúncios publicitários da marca poderão não encaixar-se no estereótipo tradicional do culto do corpo (Ribeiro, 2011).

8. Doença crónica que afecta a natural pigmentação da pele.

9. Fonte: www.movenoticias.com/2014/07/desigual-assina-contrato-com-modelo-comvitiligo/. 
E se Mota-Ribeiro (2010) encara a publicidade como uma área central na qual os discursos de género são comunicados nas sociedades contemporâneas, sustentando a respectiva desigualdade e hierarquia, a autora considera, igualmente, que se tratam de espaços privilegiados onde a mudança e a contestação de significados podem ocorrer. De facto, a publicidade apresenta uma dupla faceta, funcionando, simultaneamente, enquanto um discurso de reprodução e de transformação. A dimensão reflexiva surge quando, por exemplo, espelha estereótipos, dando-lhes continuidade e consolidando-os. Tal como escreve Cerqueira: "A publicidade tem acompanhado as mudanças sociais, mas continua a recorrer a visões limitadoras e muitas vezes estereotipadas no que concerne ao género" (2014: 8). A autora sublinha que investigações no campo publicitário apontam para o facto de a estereotipia permanecer persistente. Goffman (1979) (citado por Magalhães, 2014) argumenta que a publicidade influencia a forma como se constroem os ideais genderizados do que é a masculinidade e feminilidade.

Magalhães (2014) coloca a tónica na necessidade de compreender e explicitar os mecanismos sociais que mantêm os pressupostos usados e veiculados pela publicidade, como os estereótipos, já que está em causa um processo de influência multideterminado. No entanto, esta variável de comunicação também detém a capacidade para desconstruir concepções, numa ruptura com conceptualizações já enraizadas, dado o seu potencial interventivo. Portanto, a publicidade tanto espelha as dinâmicas sociais, funcionando como um barómetro socio-cultural, económico e político (Cerqueira, 2014), como as altera, agindo como uma força influenciadora, enquanto agente de construção social da realidade (Rosales, 2002) .

A prevalência de uma representação corporal feminina normativa na publicidade poderá ter como móbil o facto de ser importante optar-se por símbolos societais familiares, por promoverem, mais facilmente, a adesão aos produtos e serviços anunciados. Logo, preferem-se figuras entendidas como sendo 'normais', no sentido em que a maioria das pessoas (re)conhece, por já se encontrarem habituadas a esse registo, nomeadamente visual. Desta forma, o impacto criado junto do público-alvo poderá ser, potencialmente, mais mobilizador, dada a identificação promovida através do recurso a referências estéticas já assimiladas, valorizadas, interiorizadas e enraizadas, tais como os padrões de beleza ocidental. Desta forma, o uso de construções, narrativas e representações cristalizadas facilitaria a compreensão da mensagem publi- 
citária (Araújo, 2013). Frascara (1999, citado por Medeiros, 2004) afirma que os estereótipos ajudam a comunicação a ser eficaz, ao assegurarem que o processo de construção de significados pelo público-alvo não concede muito espaço a interpretações. Como consequência, poderá tornar-se mais fácil controlar a forma como o anúncio será desconstruído.

Realce-se que também o corpo masculino é submetido a um escrutínio visual, ainda que de forma assimétrica, quando comparado com o universo feminino. Curiosamente, se dois dos vectores alocados à perfeição corporal, a beleza e a juventude, são transversais aos indivíduos, independentemente do seu género (Dittmar, 2008; Veríssimo, 2008; Pereira et al., 2011), já a magreza só é associada ao corpo da mulher. No caso masculino, destaca-se um corpo musculado (Pereira et al., 2011). Veríssimo (2008) assinala que nos anúncios publicitários que analisou evidenciou-se o vigor físico e sexual das personagens masculinas, sendo mais valorizados o peito e os ombros largos, ao representarem a metáfora do triunfo profissional e sucesso. O sexo masculino começa a prestar uma cada vez maior atenção às questões estéticas (Dittmar, 2008; Mota-Ribeiro, 2010; Pereira et al. 2011).

\section{CONCLUSÃo}

A corporeidade apresenta uma particular importância na contemporaneidade, especialmente num contexto feminino. Trata-se de um processo bilateral: o corpo é, para a mulher, crucial na sua vivência quotidiana, centralidade essa igualmente reconhecida pela restante sociedade. A perfeição corporal feminina encerra uma trilogia: beleza, magreza e juventude. Este é o padrão visual dominante, o qual, teoricamente, despoletará a 'normalização' do indivíduo, através da adesão a uma normatividade estética. Para o efeito, recorrem-se às mais diversas práticas corporais, com o intuito de se obter a figura almejada. Estão em causa ideais de beleza interiorizados pelos consumidores e prioritizados pela publicidade, ao explorar dimensões aspiracionais, através de personagens que espelham corpos 'ideais', em detrimento de 'reais'. A representação corporal presente no discurso publicitário retrata, assim, uma aparência hegemónica, num discurso consentâneo com uma modelação imagética. Ainda que algumas marcas optem por comunicar novas visualidades, num discurso disruptivo para com uma estética massificada, tratamse de casos pontuais, pelo que pouco espaço é dado à pluralização visual. 
Evidencia-se a menor visibilidade de corpos 'imperfeitos': idosos, obesos, feios, doentes e com deficiência. Esperamos que o nosso artigo possa ter facultado, de alguma forma, pistas para futuras reflexões e discussões da problemática, cuja preponderância na actualidade é incontornável, pelo que urge existir um maior escrutínio sobre uma temática com repercussões directas na vivência dos indivíduos.

\section{BIBLIOGRAFIA}

Agnew, R. (1984). The effect of appearance on personality and behavior: are the beautiful really good?. Youth e Society, 15(3). Consultado em: http://yas.sagepub.com/content/15/3/285

Almeida, M. \& Tracy, K. (2003). Noites nômades: espaço e subjectividade nas culturas jovens contemporâneas. Rio de Janeiro: Rocco.

Alvarado, M. (2006). El mito de la eterna juventud: productos de belleza destinados a jóvenes. In Cardoso et al. (org.). Jovens, marcas e estilos de vida (pp. 299-308). Porto: Edições Universidade Fernando Pessoa.

Araújo, S. (2013). Príncipe ou sapo? Comparando os estereótipos masculinos na publicidade televisiva brasileira e portuguesa. Estudos em Comunicação, (13): 261-280. Covilhã: Universidade da Beira Interior. Consultado em: www.ec.ubi.pt/ec/13/pdf/EC13-2013Junho-10.pdf

Ashikali, E.-M. \& Dittmar, H. (2012). The effect of priming materialism on women's responses to thin-ideal media. British Journal of Social Psychology, 51: 514-533.

Autio, M. et al. (2013). Wealthy men and beautiful women? Constructing gender identity through consumption. In Jentl \& Kaskinen (ed.). To be young! Youth and the future, comunicação apresentada na conferência "To be Young! Youth and the Future", Finland Futures Research Centre, 6-8 de Junho de 2012. Turku, Finlândia, 133-143.

Bajoit, G. (2006). O papel da confiança na formação da identidade e do vínculo social. In Balsa (org.). Confiança e laço social (pp. 61-75). Lisboa: Edições Colibri.

Belleau et al. (2007). Theory of reasoned action: purchase intention of young consumers. Clothing and Textiles Research Journal, 25(3). Consultado em: http://ctr.sagepub.com/content/25/3/244.full.pdf 
Bessenoff, G. (2006). Can the media affect us? Social comparison, selfdiscrepancy, and the thin ideal. Psychology of Women Quarterly, (30): 239-251.

Boëthius, U. (1995). Controlled pleasures: youth and literacy texts. In Forñas \& Bolin (ed.), Youth culture in late modernity (145-168). Londres: Sage Publications.

Brinkman et al. (2012). Children's gender identity development: the dynamic negotiation process between conformity and authenticity. Youth Society, XX(X). Consultado em: http://yas.sagepub.com/content/early/2012/07/2 6/0044118X12455025.full.pdf+html

Brull, M. \& Abeja, D. (2006) El fenómeno dinki, un nuevo estilo de vida, lo último en target publicitario. In Cardoso et al. (org.). Jovens, marcas e estilos de vida (pp. 75-84). Porto: Edições Universidade Fernando Pessoa.

Buhrmester, D. \& Chow, C. (2009). Friendships in adolescence. In Reis H. \& Sprecher, S. (eds.). Encyclopedia of human relationships (pp. 718-721). Thousand Oaks: SAGE Publications. Consultado em: http://sk.sagepub. com/reference/humanrelationships/n230.xml

Camargo, H. (2013). O filme publicitário como mito atualizado: fantasia, ritual, tempo e totemismo. Biblioteca on-line de Ciências da Comunicação. Covilhã: Universidade da Beira Interior.

Campbell, C. (2004). I shop therefore I know that I am: the metaphysical basis of modern consumerism. In Ekström \& Brembeck (ed.). Elusive consumption in retrospect: report from the conference (pp. 10-21), CFK Rapport.

Campos, R. (2010). Juventude e visualidade no mundo contemporâneo: uma reflexão em torno da imagem nas culturas juvenis. Sociologia, Problemas e Práticas, (63): 113-137.

Cardoso, P. \& Pinto, S. (2009). Consumo hedónico e utilitário e atitude face à publicidade. Comunicação pública: Revista multidisciplinar de comunicação, 4(8): 99-117. Lisboa: Gabinete de Comunicação e Serviço de Informação e Documentação da Escola Superior de Comunicação Social.

Cerqueira, C. (2014). Os média, os públicos e os discursos de género: (in)visibilidades, linguagens e protagonistas. In Jorge, Sequeira \& Magalhães. 
PubliDiversidade. Representações sociais e igualdade de género na publicidade (7-16). Braga: União de Mulheres Alternativa e Resposta. Consultado em: http://repositorium.sdum.uminho.pt/bitstream/1822/381 06/1/CC_Representa\%c3\%a7\%c3\%b5es-Sociais-G\%c3\%a9nero-Public idade.pdf

Chan, K. (2008). Social comparison of material possessions among adolescents. Qualitative market research: an international journal, 11(3): 316330 .

Chang et al. (2014). Consumers with disabilities: a qualitative exploration of clothing selection and use among female college students. Clothing and Textiles Research Journal, 32(1). Consultado em: http://ctr.sagepub.com /content/32/1/34.full.pdf+html

Chidid, I. \& Leão, A. (2011). Atividades de consumo como recursos da construção da identidade pré-adolescente em interações verbais. Revista Organizações em contexto (Online), 7(13): 59-83.

Corrigan, P. (1997). The sociology of consumption: an introduction. Londres: Sage Publications.

Crymble, S. (2012). Contradiction sells: feminine complexity and gender identity dissonance in Magazine Advertising. Journal of Communication Inquiry, 3(1): 62-84.

Daters, C. (1986). A comparison of the importance of clothing and selfesteem by female adolescents from a metropolitan school district. ETD collection for University of Nebraska: Lincoln.

Dens, N. et al. (2008) Effects of nudity in advertising on consumers' body esteem. In Pereira et al. (ed.). New trends in advertising research (pp. 415-427). Lisboa: Edições Sílabo.

Deutsch, N. \& Theodorou, E. (2010). Aspiring, consuming, becoming: youth identity in a culture of consumption. Youth e Society, 42(2). Consultado em: http://yas.sagepub.com/content/42/2/229.full.pdf+html

Dittmar, H. (2008). Consumer culture, identity and well-being: the searche for "good life" and the "body perfect". Londres: Psychology Press.

Dittmar, H. (2009). How do "body perfect"ideals in the media have a negative impact on body image and behaviors? Factors and processes related to self and identity. Journal of Social and Clinical Psychology, 28(1): 1-8. 
Dittmar, H. et al. (2007). When a better self is only a button click away: associations between materialistic values, emotional and identity related buying motives, and compulsive buying tendency online. Journal of Social and Clinical Psychology, 26(3): 334-361.

Fannon, T. (2016). Out of sight, still in mind: visually impaired women's embodied accounts of ideal femininity. Revista Disability Studies Quarterly, 36(1). Consultado em: http://dsq-sds.org/article/view/4326/4210

Fehr, B. (2009). Friendship formation and development. Encyclopedia of human relationships. Sage Publications.

Featherstone, M. (1994). Consumer culture e postmodernism. Londres: Sage Publications.

Featherstone, M. (1991) The body in consumer culture. In Featherstone et al. (ed.). The body: social process and cultural theory (pp.170-196). Londres: Sage Publications.

Ferreira, P. (2000). Controlo e identidade: a não conformidade durante a adolescência. Sociologia, Problemas e Práticas, 33: 55-85.

Ferreira, V. (2003). Atitudes dos jovens portugueses perante o corpo. In Pais \& Cabral (coord.). Condutas de risco, práticas culturais e atitudes perante o corpo: resultados de um inquérito aos jovens portugueses em 2000 (pp. 265-366). Oeiras: Celta.

Ferreira, V. (2004). Da reflexividade corporal entre os jovens portugueses: uma realidade socialmente fragmentada. Actas dos ateliers do V Congresso Português de Sociologia, Sociedades Contemporâneas: Reflexividade e Acção. Atelier Corpo e sexualidade. Consultado em: www.aps.pt /cms/docs_prv/docs/DPR4616d905aae8b_1.pdf

Ferreira, V. (2011). Dar corpo à juventude: o corpo jovem e os jovens nos seus corpos. In Bendit, Pais \& Ferreira (orgs.), Jovens e Rumos (pp. 257-276). Lisboa: Imprensa de Ciências Sociais. Consultado em: http://vitorsergio ferreira.net/wp-content/uploads/2014/12/2011-Dar-corpo-\%C3\%A0-juv entude-Jovens-e-Rumos.pdf

Fonseca, R. (2007). A arte como discurso: a identidade como mercadoria. Sociologia, Problemas e Práticas, (53): 117-133. 
Ganetz, H. (1995). The shop, the home and femininity as a masquerade. in Forñas \& Bolin (ed.). Youth culture in late modernity (pp. 72-99) Londres: Sage Publications.

Gay, P. et al. (1997). Doing cultural studies: the story of the Sony Walkman. Londres: Sage Publications.

Gonçalves, A. (1995). Construção social de identidades juvenis em contexto de exclusão social. Dissertação de Mestrado em Sociologia do Território. Lisboa: ISCTE.

Gove, J. \& Watt, S. (2004). Identity and gender. In Woodward (ed.). Questioning identity: gender class, ethnicity (pp. 43-77). Londres: Routledge.

Green, L. (2004). Gender. In Taylor \& Spencer (ed.), Social identities: multidisciplinary approaches (pp.35-63). Nova Iorque: Routledge.

Gregson, K. (2006). Youth culture. Encyclopedia of children, adolescents, and the media. Sage Publications.

Gunter, B. \& Furnham, A. (1998). Children as consumers: a psychological analysis of the young people's market. Londres: Routledge. Consultado em: http://yas.sagepub.com/content/early/2012/07/26/0044118X124550 25

Haller, B. \& Ralph, S. (2001). Profitability, diversity, and disability images in advertising in the United States and Great Britain. Revista Disability Studies Quarterly, 21(2). Consultado em: http://dsq-sds.org/article/view/27 $6 / 301$

Halliwell, E. \& Dittmar, H. (2004). Does size matter? the impact of model's body size on women's body-focused anxiety and advertising effectiveness. Journal of Social and Clinical Psychology, 23(1): 104-122.

Henderson, B. \& DeLong, M. (2000). Dress in a postmodern era: an analysis of aesthetic expression and motivation. Clothing and Textiles Research Journal, 18(4). Consultado em: http://ctr.sagepub.com/cgi/content/abstr act/18/4/237

Hockey, J. \& James, A. (2003). Social identities across the life course. Hampshire: Palgrave Macmillan.

Holdorf, R. (2005). The role of teen oriented fashion magazine content on clothing purchase behaviors and attitudes of adolescents and teens. Florida State University D-Scholarship Repository. Consultado em: http://di 
gitool.fcla.edu/view/action/singleViewer.do?dvs=1346930142331606el ocale=pteVIEWER_URL=/view/action/singleViewer.do?eDELIVERY_ RULE_ID=7eadjacency=Neapplication=DIGITOOL-3eframeId=1euse $\mathrm{Pid} 1=$ trueeusePid2=true

Hosltein-Beck, S. (1995). Consistency and change in the lifeworld of young women. In Forñas \& Bolin (ed.), Youth culture in late modernity (pp. 100-119). Londres: Sage Publications.

Januário, S. \& Cascais, A. (2012), O corpo masculino na Publicidade: uma discussão contemporânea. Revista Comunicação e Sociedade, 21: 135148.

Jorge, A. (2014). "Mas se na sociedade também é assim ...”. Perceções face às representações de género na publicidade... In Jorge, Sequeira \& Magalhães. PubliDiversidade. Representações sociais e igualdade de género na publicidade (35-54). Braga: União de Mulheres Alternativa e Resposta. Consultado em: http://repositorium.sdum.uminho.pt/bitstream/18 22/38106/1/CC_Representa\%c3\%a7\%c3\%b5es-Sociais-G\%c3\%a9nero -Publicidade.pdf

Lazar, M. (2012), Género, guerra e políticas do corpo: uma análise crítica multimodal da metáfora na Publicidade. Comunicação e Sociedade, (19): 183-203, Braga: Centro de Estudos de Comunicação e Sociedade, Universidade do Minho. Consultado em: https://repositorium.sdum.uminho. pt/bitstream/1822/29818/1/Revista_Comunicacao_e_Sociedade_21.pdf

Lehmann, L. et al. (1998). Estetização do corpo: identificação e pertencimento na contemporaneidade. In Castro (org.). Infância e adolescência na cultura do consumo (pp.107-123), Rio de Janeiro, Nau.

Maffesoli, M. (1990). A física mística do corpo. Revista de comunicação e linguagens: o corpo, o nome, a escrita, 10/11: 33-40. Lisboa: Centro de Estudos de Comunicação e Linguagens, Faculdade de Ciências Sociais e Humanas.

Magalhães, S. (2014). Análise de anúncios publicitários em revistas estilo de vida portuguesas - Um estudo de caso. In Jorge, Sequeira \& Magalhães. PubliDiversidade. Representações sociais e igualdade de género na publicidade (17-34). Braga: União de Mulheres Alternativa e Resposta. Consultado em: http://repositorium.sdum.uminho.pt/bitstream/1822/381 
06/1/CC_Representa\%c3\%a7\%c3\%b5es-Sociais-G\%c3\%a9nero-Public idade.pdf

Magalhães, S. (2011). Como ser uma Ragazza. Discursos de sexualidade numa revista para raparigas adolescentes. Dissertação de Doutoramento em Psicologia. Braga: Universidade do Minho. Consultado em: http://re positorium.sdum.uminho.pt/bitstream/1822/19651/1/Sara\%2

OIsabel\%20Almeida\%20Magalh\%c3\%a3es.pdf

Marion, G. \& Nairn, A. (2011). We make the shoes, you make the story. Teenage girl's experiences of fashion: Bricolage tactics and narrative identity. Consumption, Markets e Culture, 14(1): 29-56.

Medeiros, C. (2001). Personagens, espaços e cenários na publicidade. In Pereira, F. \& Veríssimo, J. (coord.). Publicidade, o estado da arte em Portugal (pp. 73-91). Lisboa: Edições Sílabo.

Mota-Ribeiro, S. (2003). Corpos visuais: imagens do feminino na publicidade. In Macedo, A. \& Grossegesse, O. (org.). Re-presentações do corpo (pp. 115-132). Braga: Centro de Estudos Humanísticos da Universidade do Minho. Consultado em: http://repositorium.sdum.uminho.pt/handle/1 $822 / 5309$

Mota-Ribeiro, S. (2010). Do outro lado do espelho: Imagens e discursos de género nos anúncios das revistas femininas - uma abordagem sociosemiótica visual feminista. Dissertação de Doutoramento em Ciências da Comunicação, Especialidade de Semiótica Social. Braga: Universidade do Minho. Consultado em: http://repositorium.sdum.uminho.pt/handle/1 $822 / 12384$

Nielsen, H. \& Rudberg, M. (1993). Gender, body and beauty in adolescence: Three psychological portraits. Young, 1(2). Consultado em: http://you.sagepub.com/content/1/2/32

Nunes, B. (2007). Consumo e identidade no meio juvenil: considerações a partir de uma área popular do Distrito Federal. Sociedade e Estado, 22(3): 647-678.

Padilla-Walker, L. (2006). Adolescents, developmental needs of, and media. Encyclopedia of children, adolescents, and the media. Sage Publications.

Pais, J. (1995). Éticas e estéticas do quotidiano. In Santos (coord.), Cultura e Economia. Actas do Colóquio realizado em Lisboa, 9-11 de Novembro 
de 1994 (pp.129-152). Lisboa: Instituto de Ciências Sociais da Universidade de Lisboa.

Pais, J. (2003). Grupos juvenis: condutas e imagens. In Pais \& Cabral (coord.). Condutas de risco, práticas culturais e atitudes perante o corpo: resultados de um inquérito aos jovens portugueses em 2000 (pp. 367412). Oeiras: Celta.

Pais, J. (2005). Jovens e cidadania. Sociologia, Problemas e Prácticas, (49): $53-70$.

Pais, J. (2010). Lufa-lufa quotidiana: ensaios sobre cidade, cultura e vida urbana. Lisboa: ICS - Imprensa de Ciências Sociais.

Pais, J. (1996). Vivências sexuais: modos e diversidades. III Congresso Português de Sociologia, Práticas e Processos da Mudança Social. Consultado em: http://www.aps.pt/cms/docs_prv/docs/DPR492ede2664249_1. pdf

Palacios, A. (2004. As marcas na pele, as marcas no texto. Sentidos de tempo, juventude e saúde na publicidade de cosméticos em revistas femininas durante a década de 90. Pós-Graduação em Comunicação e Cultura Contemporâneas. Universidade Federal da Bahia. Consultado em: www.bocc.ubi.pt/pag/palacios-annamaria-marcas-na-pele.pdf

Pereira, F. \& Antunes, A. (2008). Trends in cosmetics advertising in Portugal. In Pereira et al. (ed.), New trends in advertising research (pp. 373-389). Lisboa: Edições Sílabo.

Pereira, F. \& Veríssimo, J. (coord.) (2004). Publicidade, o estado da arte em Portugal. Lisboa: Edições Sílabo.

Pereira, F. et al. (2005). Consumo e auto-estima. Comunicação Pública: Revista multidisciplinar de comunicação, 1(1): 135-157. Lisboa: Gabinete de Comunicação e Serviço de Informação e Documentação da Escola Superior de Comunicação Social.

Pereira, F. et al. (2011). O papel da publicidade na compra de produtos cosméticos. Comunicação e Sociedade, (19): 161-178, Braga: Centro de Estudos de Comunicação e Sociedade, Universidade do Minho.

Perse, E. (2006). Advertising, effects on adolescents. Encyclopedia of children, adolescents, and the media. Sage Publications. 
Pinto-Coelho \& Mota-Ribeiro (2012). Analisando os discursos de género das e sobre as imagens publicitárias de mulheres: como articular a análise sociosemiótica com a análise da recepção. Biblioteca on-line de Ciências da Comunicação. Covilhã: Universidade da Beira Interior. Consultado em: www.bocc.ubi.pt/pag/zara-pinto-coelho-silvanamota-ribeiro-discur sos-de-genero.pdf.

Pinto-Coelho \& Mota-Ribeiro (2012). O reino do casal heterossexual na publicidade: uma análise sociosemiótica das estratégias visuais e inscrições discursivas. Comunicação e Sociedade, (19): 161-178. Braga: Centro de Estudos de Comunicação e Sociedade, Universidade do Minho. Consultado em: http://revistacomsoc.pt/index.php/comsoc/article/view/709.

Pons, A. (2006). Los arquetipos cinematográficos, modelos para la publicidad y creadores de valores sociales. In Cardoso et al. (org.), Jovens, marcas e estilos de vida (pp. 309-316). Porto: Edições Universidade Fernando Pessoa.

Reimer, B. (1995). The media in public and private spheres. In Forñas \& Bolin (ed.), Youth culture in late modernity (pp. 58-71). Londres: Sage Publications.

Ribeiro, M. (2011). Beleza feminina e publicidade: um estudo sobre as campanhas da marca Dove. Dissertação de Doutoramento em Comunicação Social. Porto Alegre: Pontifícia Universidade Católica do Rio Grande do Sul. Consultado em: http://repositorio.pucrs.br/dspace/handle/10923/22 34.

Ribeiro, R. (2010). Sociologia do consumo: aplicado ao marketing e à comunicação. Lisboa: Instituto Superior de Ciências Sociais e Políticas.

Ritzer, G. (2005). Enchanting a disenchanted world: revolutionizing the means of consumption. California: Pine Forge Press.

Rodrigues, E. (2013). Dar corpo ao género: uma análise sociológica das masculinidades. Dissertação de Doutoramento em Sociologia. Lisboa: ISCTE.

Rosales, M. (2002). Temos o que procura. Coimbra: Minerva.

Santos, C. (2012). O poder simbólico do consumo: no trilho da génese do discurso publicitário. Revista Comunicando, SOPCOM, 1(1): 29-42. 
Santos, C. (2013). Publicidade e identidade: que relação?. Revista Comunicação Pública, 8(14): 37-55. Lisboa: Escola Superior de Comunicação Social.

Schmidt, L. (1989). O discurso publicitário e a construção da juventude como categoria social. Lisboa: Instituto de Ciências Sociais da Universidade de Lisboa.

Silva, C. (org.) (2001). Os jovens, o dinheiro e o crédito. Lisboa: Instituto do Consumidor.

Silva, R. (2011). Valores e felicidade no Séc. XXI: um retrato sociológico dos portugueses em comparação europeia. Dissertação de Doutoramento em Sociologia. Lisboa: ISCTE-IUL.

Simmel, G. (1969). Cultura feminina. Alfragide: Galeria Panorama.

Simmel, G. (1991). Fashion. In Ray (ed.), Formal Sociology: the sociology of Georg Simmel. Aldershot: Edward Elgar Publishing.

Simmel, G. (2004). Fidelidade e gratidão e outros textos. Lisboa: Relógio D'Água Editores.

Strandbu, A. \& Kvalem, I. (2014). Body talk and body ideals among adolescent boys and girls: a mixed-gender focus group study. Youth e Society, (46): 623-641.

Twigg, J. (2009). Clothing, identity and the embodiment of age. In Powell \& Gilbert (ed.) Aging and identity: a postmodern dialogue. Nova Iorque: Nova Science Publishers.

Veríssimo, J. (2008). O corpo na publicidade. Lisboa: Edições Colibri/Instituto Politécnico de Lisboa.

Vilhjalmsson, R. (2012). Bodily deviations and body image in adolescence. Youth e Society, 44(3): 366-384. 